\title{
8 Zusammenfassung
}

Regionalorganisationen haben einen wichtigen Stellenwert in nahezu allen Politikfeldern. An Zahl und Bedeutung haben sie nach dem Ende des Kalten Krieges zugenommen. In der akademischen Debatte über Regionalorganisationen, und den dahinterliegenden Prozessen der Regionalisierung bzw. des region-building, bleiben zahlreiche Herausforderungen für die wissenschaftliche Auseinandersetzung mit diesem Phänomen bestehen. Dies gilt mit Blick auf die aktive Rolle von Regionalorganisationen in gegenwärtigen Globalisierungsprozessen, die Periodisierung von Regionalismen über die vergangenen 200 Jahre sowie die methodologischen Parameter einer vergleichenden Erforschung von Regionalismen und Regionalorganisationen. Empirisch besteht nach wie vor großer Bedarf an detaillierten Fallstudien über die internen Dynamiken und Entscheidungsprozesse sowohl innerhalb von Regionalorganisationen wie auch mit Blick auf InterRegionalismen. Dies gilt in zahlreichen Fällen auch für die Rekonstruktion der oftmals kaum dokumentierten nationalen Interessen in Regionalorganisationen, dem Zusammenspiel von staatlichen und nicht-staatlichen Akteuren oder den Finanzierungsregimes von Regionalorganisationen. ${ }^{1}$ Die Akteursqualität von Regionalorganisationen ist mittlerweile weitgehend unbestritten, jedoch mangelt es weiterhin an Studien, die dies empirisch breit abgesichert und Politikfeld-bezogen sowohl gegenüber den jeweiligen Mitgliedsstaaten wie auch gegenüber dem „internationalen System“ und anderen Regionalorganisationen untersuchen. Und schließlich bleibt noch gewaltig Raum, um die Professionalisierung und Institutionalisierung von Regionalorganisationen im Globalen Süden, deren interne Lernprozesse sowie die kulturellen Transfers zwischen verschiedenen Regionalorganisationen zu untersuchen.

Die Darstellung der verschiedenen Debatten über Regionalorganisationen, Regionalismen und Regionen sowie die empirischen Beispiele aus der Afrikanischen Union sollten verdeutlichen, worin der analytische Mehrwert der Konzepte Raumformat und Raumordnung, wie sie in dem vorliegenden Beitrag skizziert worden sind, liegen kann. Indem in Fortsetzung eines post-strukturalistischen Raumverständnisses der Blick auf die Signifizierung von Raumimaginationen konkreter Akteure in intersubjektiven Prozessen gerichtet wird, lassen sich die verwendeten Raum-Schablonen, -Modelle, -Muster oder -Vorbilder im Detail in ihrer Historizität und Entstehung sowie in ihrem Verhältnis zu anderen, konkurrierenden Raumformaten analysieren. Raumformate sind eine Linse, durch die

1 Zum letztgenannten Punkt vgl. U. Engel and F. Mattheis (eds.), Follow The Money. The Finances of Regional Security Organisations in Africa, Asia and Latin America, Abingdon: Routledge (i.E.). 
Räume von Raum-Entrepreneuren und unter Zuhilfenahme von Bedeutungsaufladungen einem bestimmten Resonanzpublikum gegenüber kommuniziert werden. Beim Ordnen von Räumen werden Vorstellungen von der „Adäquatheit“, „Relevanz“ und „Angemessenheit“ bestimmter Raumformate verhandelt. Der Prozess der Sichtbarmachung von „gültigen“ Raumformaten und der Herstellung von intersubjektiv geteilten Raumordnungen, also die Praxis des Raumordnens, ist zentral, um zu verstehen, wie „soziale Räume“ konstituiert werden und wie sie sich in Vergangenheit und Gegenwart zu einander verhalten. Die „Region“ bleibt dabei eines der bedeutsamsten Raumformate zum Verständnis aktueller Globalisierungsprozesse. 
